\title{
Associative priming in symbolic comparisons by adults and children
}

\author{
MARC MARSCHARK \\ University of North Carolina at Greensboro, Greensboro, North Carolina \\ MARGARITA AZMITIA \\ University of Minnesota, Minneapolis, Minnesota \\ and \\ ALLAN PAIVIO \\ University of Western Ontario, London, Ontario, Canada
}

\begin{abstract}
Two experiments examined larger and smaller judgments of animal and object word pairs. In the pilot experiment, we used the standard tachistoscopic paradigm; in the second experiment, we employed a modified pencil-and-paper version. Congruity effects (i.e., comparative $\times$ relative magnitude interactions) were consistently larger with animal than with object stimuli across subjects ranging from second graders to university students. These results support a semantic priming interpretation of the congruity effect and emphasize the need to consider the retrieval component as well as the comparison stage in symbolic judgments.
\end{abstract}

Studies of how people mentally compare relative magnitudes have contributed to several recent advances in understanding human cognition and memory. For the most part, interest has focused on the comparison stage of judgments, wherein reaction times are found to decrease as the rated difference between the stimuli increases. Relatively little attention, however, has been given to the retrieval component of the comparison task.

The importance of retrieval in symbolic comparisons is suggested by several recent findings concerning the congruity effect, an interaction between comparative and relative magnitude such that, for example, subjects are faster in choosing the larger of two large stimuli and the smaller of two small stimuli than in choosing the smaller of two large stimuli or the larger of two small stimuli. In particular, two studies have demonstrated that congruity effects are obtained in symbolic (memorial) comparisons but not in purely perceptual comparisons (Banks, Mermelstein, \& Yu, 1982; Marschark \& Paivio, 1981). These results appear to pose a problem for researchers assuming that memorial and perceptual comparisons are directly analogous (e.g., Moyer, 1973). However, it is unclear whether the observed divergence is a function of differences in the comparisons per se or in the nature of the stimuli; that is, the presence and absence of congruity effects could be a consequence of differences in discriminating percep-

This research was facilitated by a grant to Marc Marschark from the Research Council of the University of North Carolina at Greensboro and Grant A0087 to Allan Paivio from The Natural Science and Engineering Research Council of Canada. Correspondence should be addressed to Marc Marschark, Department of Psychology, University of North Carolina at Greensboro, Greensboro, NC 27412. tual information versus internally generated information (e.g., Holyoak \& Mah, 1982) or of the fact that only symbolic judgments involve prior memorial activation (Marschark \& Paivio, 1981; see Marschark, 1983a, for elaboration).

On the basis of Marschark and Paivio's (1981) perceptual comparison findings noted above, they distinguished the comparison stage of the judgment task, which produces symbolic distance effects, from the informationretrieval stage, which produces the congruity effect (Duncan \& McFarland, 1980). According to Marschark and Paivio's explanation, each comparative in the comparative-then-stimulus paradigm activates a subset of memorial information congruent with it (e.g., presentation of "smaller" may initiate spreading activation of the size attributes for relatively small animals). If the following stimuli are congruent and associated with the comparative (Marschark, 1983b), they would have a relatively high probability of being primed, and a fast response would be obtained. Incongruent stimuli, in contrast, would slow the response due to the need to redirect the search for magnitude information relevant to them. Over a series of such trials, a congruity effect would be obtained.

\section{PILOT STUDY}

Any priming or activation model of a memory process must be able to accommodate the categorical structure of memory. Specifically, priming within a single category should be faster or more comprehensive than priming across categories, and a "prime" such as choose the larger item should produce relatively greater benefits within a restricted stimulus set than within an unrestricted 
set. Thus, if priming is the mechanism of congruity effects, those effects should be larger in cases characterized by a greater specificity of (correct and incorrect) activation (cf. Banks \& Flora, 1977; Holyoak \& Mah, 1982).

This hypothesis was initially tested using a tachistoscopic comparative-stimulus judgment paradigm. Half of the 40 subjects received a stimulus set composed of 120 animal pairs, and the other subjects received a set composed of 60 animal pairs and 60 nonanimal pairs (see Marschark \& Paivio, 1981, for a description of the standard methodology).

Although the animal and mixed lists did not differ significantly in overall reaction times, list type did interact with the congruity effect (i.e., a reliable list type $\times$ comparative $\times$ relative magnitude interaction). The magnitudes of the congruity effects in the animal and mixed list conditions were 48 and $24 \mathrm{msec}$, respectively. ${ }^{1}$ These results clearly supported the prediction from the semantic priming interpretation of congruity effects; nevertheless, because of the potential importance of these findings, the study was replicated in the following experiment. A modified pencil-and-paper methodology was employed so that the task could be extended to include second- and sixthgrade children as well as high school and college students.

Several recent studies have shown that, by age 7, children already have an extremely complex and stable organization of long-term memory. This structure is based, at least in part, on categorical and associative relationships of the sort examined in the pilot experiment, relationships which have been shown to be functionally automatized by this age (e.g., McCauley, Weil, \& Sperber, 1976). Given young children's dexterity in activating both categorical and associative knowledge, the bipolar comparison task seemed a useful tool for examining the development of both memory organization and semantic priming.

\section{METHOD}

\section{Subjects}

The second- and sixth-grade samples were composed of 20 subjects each. The mean age of the second graders was 8 years, 0 months, and of the sixth graders 11 years, 7 months. The 20 high school subjects were sophomores and juniors enrolled in a psychology course for gifted and talented students taught by the first author. The 60 university subjects were introductory psychology volunteers.

\footnotetext{
Stimuli and Design

Eight lists of 25 stimulus pairs each were constructed, four composed of animal names and four of object names. Two lists of each type contained names of things relatively large in real-world size, and two lists contained names of things relatively small, according to available norms. Animal and object stimuli were equally extreme in their relative magnitudes and appeared equally familiar. The lists were constructed so that the rated differences between the sizes of the members of the pairs were approximately equal across lists and large enough to allow unambiguous decisions even by the second graders. Stimulus lists were printed, double spaced and in capitals, on separate sheets of paper and were compiled into test booklets. Lists were paired with the comparatives "smaller" and "larger" to yield a counterbalanced 2 (animals or objects) $\times 2$ (relative magnitude: large or small) $\times 2$ (comparative: "larger" or "smaller") design for each sample.
}

\section{Procedure}

Second- and sixth-grade subjects were tested individually. They each were told that they were going to be shown some lists with animal words and some with "other things." Sometimes the experimenter would ask them to circle the word in each pair that would be larger if the two were seen together and sometimes the thing that would be smaller. After each comparative was given, the subject turned over a test page and circled the items as fast as possible in $60 \mathrm{sec}$, being careful not to make mistakes. The older samples received similar instructions but were tested in groups and were given $30 \mathrm{sec}$ per list.

\section{RESULTS}

Although all stimuli were assumed to be familiar to even the youngest subjects, analyses of the error data provided a means of externally validating the appropriateness of their selection. Because there was no consistent pattern to the error data, it appeared that all stimuli were of approximately equal difficulty for all subjects.

Mean (per pair) correct reaction times were calculated for each list of each subject and were analyzed using an analysis of variance corresponding to the above design. Unless otherwise noted, all and only those effects reported below were reliable at or beyond the .05 level. These data are presented in Table 1, where it can be seen that reaction times generally decreased with grade $[\mathrm{F}(3,116)=$ $691.56, \mathrm{MSe}=3.18]$. The overall effect of comparative was reliable ("larger" judgments were faster than "smaller" judgments) $[\mathrm{F}(1,116)=10.46, \mathrm{MSe}=2.31]$ and varied with grade $[\mathrm{F}(3,116)=11.67]$. The overall congruity effect (the comparative $\times$ relative magnitude interaction) also was reliable $[\mathrm{F}(1,116)=10.70, \mathrm{MSe}=$ 3.98 , and the effect was larger for animal than for object stimuli $[\mathrm{F}(1,116)=4.29, \mathrm{MSe}=5.20]$. This threeway interaction also showed developmental changes (interacting with grade) $[\mathrm{F}(3,116)=3.54]$. Finally, rela-

Table 1

Mean Comparison Times and Congruity Effects (in Seconds) for Four Age Groups

\begin{tabular}{ccccc}
\hline \multirow{2}{*}{ Relative Magnitude } & \multicolumn{2}{c}{ Smaller } & Larger & Congruity \\
Effect
\end{tabular}


tively small animals and large objects were compared faster than relatively large animals and small objects, yielding a materials $\times$ relative magnitude interaction $[\mathrm{F}(1,116)=4.89, \mathrm{MSe}=3.93]$ that also interacted with grade $[\mathrm{F}(3,116)=3.01]$.

The higher order interactions were examined further by considering separately the animal and object data. In the animal comparison data, response latencies decreased with grade $[\mathrm{F}(3,116)=444.03, \mathrm{MSe}=2.47]$, and small stimuli were compared faster than large stimuli $[\mathrm{F}(1,116)=5.64, \mathrm{MSe}=5.09]$. "Larger" judgments were made faster than "smaller" judgments $[F(1,116)=$ 4.12, $\mathrm{MSe}=4.27]$, and this varied with grade $[\mathrm{F}(3,116)=2.66, \mathrm{p}=.05]$, although Newman-Keuls analyses showed none of the individual effects to be reliable. The overall animal congruity effect was highly reliable $[\mathrm{F}(1,116)=20.05$, MSe $=3.16]$, and also varied with grade $[F(3,116)=6.61]$. Tests of the simple effects in each grade showed all of the animal congruity effects to be reliable.

Analysis of the object-comparison data also revealed a reliable decrease in response latencies with grade $[\mathrm{F}(3,116)=242.99, \mathrm{MSe}=4.54]$. Although the overall effect of comparative was not reliable $[\mathrm{F}(1,116)=$ $3.59, \mathrm{p}<.10]$, it interacted with grade $[\mathrm{F}(3,116)=$ 9.06, MSe $=2.12$ ]; Newman-Keuls analyses showed only the second graders' effect ("larger" judgments faster) to be reliable. In contrast to the animal comparison data, the overall object congruity effect was not reliable and did not vary reliably with grade [both $\mathrm{Fs}<1, \mathrm{MSe}=6.02$ ] For comparison purposes, however, tests of the simple congruity effects were done anyway. Only the university sample yielded a reliable effect $[\mathrm{F}(1,19)=7.74$, MSe $=.32]$.

\section{DISCUSSION}

The present findings indicate that categorical knowledge of to-becompared stimuli can affect the activation of associative size information in children as young as 8 years (see also McCauley et al., 1976). These findings are consistent with results of previous studies demonstrating the automatic nature of categorical encoding by children of this age, but also show that such processes can facilitate other aspects of information retrieval.

The results of both experiments also are consistent with the semantic priming interpretation of congruity effects in symbolic comparisons (Marschark, 1983a; Marschark \& Paivio, 1981). The priming position is that, in bipolar symbolic comparisons involving comparative-stimuli paradigms, the comparative term acts as a cue that activates congruent stimulus magnitude information in memory. This interdependence between comparative and stimulus processing accounts for the patterns of effects obtained in a variety of comparison tasks (see Marschark, 1983a, 1983b, for reviews). The comparable overall reaction times obtained with animal and object stimuli support the assumption that attribute information of familiar animals and objects is equally comparable. Deviations about those means in the form of congruity effects, however, indicated that the priming of attribute information was relatively more likely or more successful when there are specific limits on the range of to-be-primed stimuli.

Finally, these findings demonstrate the importance of retrieval processes in symbolic judgments. One reason for concern in this regard is that inferences previously have been made about the mental representation of attribute information on the basis of data arising only from the comparison stage in this paradigm (e.g., Moyer, 1973; Paivio, 1975). Interfacing internal (memorial) information with the external world in symbolic comparisons and other tasks, however, involves encoding, retrieval, and verification processes, none of which can be understood in isolation (Marschark, 1983a).

\section{REFERENCES}

BANKs, W., \& FloRA, J. (1977). Semantic and perceptual processes in symbolic comparisons. Journal of Experimental Psychology: Human Perception \& Performance, 3, 278-290.

Banks, W. P., Mermelstein, R., \& Yu, H. K. (1982). Discriminations among perceptual and symbolic stimuli. Memory \& Cognition, 10, 265-278.

Duncan, E. M., \& McFarland, C. E., JR. (1980). Isolating the effects of symbolic distance and semantic congruity in comparative judgments: An additive-factors analysis. Memory \& Cognition, 8, 612-622.

HOLYOAK, K., \& MAH, W. (1982). Cognitive reference points in judgments of symbolic magnitude. Cognitive Psychology, 14, 328-352.

MARSCHARK, M. (1983a). Expectancy, equilibration, and memory. In J. Yuille (Ed.), Imagery, memory, and cognition: Essays in tribute to Allan Paivio. Hillsdale, NJ: Erlbaum.

MARSCHARK, M. (1983b). Semantic congruity in symbolic comparisons: Salience, expectancy, and associative priming. Memory \& Cognition, 11, 192-199.

Marschark, M., \& Paivio, A. (1981). Congruity and the perceptual comparison task. Journal of Experimental Psychology: Human Perception \& Performance, 7, 290-308.

McCauley, C., Weil, C., \& Sperber, R. (1976). The development of memory structure as reflected by semantic priming effects. Journal of Experimental Child Psychology, 22, 511-518.

MOYER, R. (1973). Comparing objects in memory: Evidence suggesting an internal psychophysics. Perception \& Psychophysics, 13, 180-184.

Paivio, A. (1975). Mental comparisons through the mind's eye. Memory \& Cognition, 3, 635-647.

\section{NOTE}

1. The magnitudes of congruity effects were calculated according to the formula $\mathrm{CE}=[(\mathrm{ML}-\mathrm{MS})+(\mathrm{US}-\mathrm{UL})] / 4$, where $\mathrm{M}$ and $\mathrm{U}$ refer to the marked and unmarked adjectives "smaller" and "larger," respectively, and S and L refer to small and large relative magnitudes, respectively.

(Manuscript received for publication June 19, 1985.) 